\title{
Polarimetric Optimization of Temporal Sublook Coherence for DInSAR Applications
}

\author{
Rubén Iglesias, Student Member, IEEE, Dani Monells, Carlos López-Martínez, Senior Member, IEEE, Jordi J. \\ Mallorqui, Senior Member, IEEE, Xavier Fabregas, Member, IEEE, and Albert Aguasca, Member, IEEE
}

\begin{abstract}
The application of Differential SAR Interferometry (DInSAR) techniques has been traditionally limited to the singlepolarimetric case. The launch of satellites with polarimetric capabilities has triggered the synergy between polarimetric and interferometric algorithms, leading to a significant improvement in final DInSAR products [1]. During the last years, the different polarimetric optimization techniques available have been successfully applied to the so-called classical phase quality estimators, i.e., the coherence and the amplitude dispersion estimators. In this context, a new estimator to evaluate the pixels' phase quality, referred to as temporal sublook coherence (TSC), has recently demonstrated to provide promising results in DInSAR applications [2]. The nature of this estimator, which is based on exploiting the spectral properties of point-like scatterers through the coherence evaluation of different sublooks of the image spectrum, allows its adaptation to the existing polarimetric optimization methods. This paper presents the benefits of extending the TSC estimator to work with fully-polarimetric data. For this purpose, a fully-polarimetric data set consisting of ten X-Band Ground-Based SAR images is employed. The final DInSAR results obtained by means of TSC polarimetric optimization are compared with the ones obtained with its classical single-polarimetric approach, achieving up to more than a twofold increase in the pixels' density.
\end{abstract}

Index Terms-Temporal Sublook Coherence, DInSAR, GBSAR, PolDInSAR, Polarimetric Optimization.

\section{INTRODUCTION}

$\mathrm{D}$ ifferential SAR Interferometry (DInSAR) techniques have demonstrated their usefulness for the precise monitoring of complex deformation phenomena with millimetric precision. In this framework, not all the pixels within the area of study present a sufficient phase quality to be included in the DInSAR processing. For this reason, an adequate selection of high-quality pixel candidates is mandatory. Two main criteria are available for the estimation of the pixels' phase statistics: the coherence stability for the selection of high-quality distributed scatterers [3] and the amplitude dispersion for the selection of the so-called Permanent Scatterers (PS) [4]. Due to the lack of long-time polarimetric SAR (PolSAR) data, the application of these techniques has been traditionally limited

Manuscript received March x, 2014. This research work received partial support from the Big Risk project (contract number BIA2008-06614), and the project TEC2011-28201-C02-01 funded by the Spanish MICINN and FEDER funds.

Authors are with the Remote Sensing Laboratory, Universitat Politècnica de Catalunya, 08034 Barcelona, Spain (e-mail: mallorqui@tsc.upc.edu). to single-polarimetric data. The launch of polarimetric SAR sensors, such as ALOS-PALSAR, RADARSAT-2 or TerraSAR-X, has allowed the extension of DInSAR techniques to fully-polarimetric data. In this context, Polarimetric Differential SAR Interferometry (PolDInSAR) algorithms have recently demonstrated to outperform classical DInSAR techniques [1][5]-[8].

A new phase quality estimator, referred to as temporal sublook coherence (TSC), has recently applied to DInSAR purposes providing promising results [2]. The method consists on characterizing the degree of correlation between different sublooks of the image spectrum along a multi-temporal set of SAR images. Those targets exhibiting high values of TSC are directly related to as point-like scatterers and, consequently, as high-quality pixels with a low phase standard deviation [2].

This paper presents the extension of the original singlepolarization TSC approach to fully-polarimetric data. The objective is to improve the TSC performance in terms of both phase quality and pixels' density. In this framework, the polarimetric optimization methods referred to as Best [5], SubOptimum Scattering Mechanism (SOM) [9], and Equal Scattering Mechanism (ESM) [10] are adapted to work with the TSC approach.

The paper is organized as follows. Section II shows a general overview of the original TSC method. Section III presents the adaptation of polarimetric optimization methods to extend the TSC estimator to the fully-polarimetric case. Section VI describes the test-site and the polarimetric data set employed for the validation. The final DInSAR results obtained by means of TSC polarimetric optimization techniques are finally compared with the ones obtained with its original single-polarimetric approach.

\section{BASIS OF TSC ESTIMATION}

The rationale of the TSC approach is based on exploiting the spectral properties of the point-like scatterers within the area of study. The objective is to identify, along the multitemporal set of acquisitions, those targets having a highcorrelated spectrum in range, azimuth, and height [2].

Compared with traditional approaches, the TSC estimator presents clear advantages [2]. Unlike the PS approach, it is not affected by amplitude fluctuations, since the amplitude now plays no role in the detection. Unlike the coherence stability estimator, which works over multi-looked interferograms, the resolution of the images is better preserved. Only a loss of a 
factor of two in the range direction is produced due to the sublook generation process. Finally, reliable full-resolution estimations of the phase statistics may be retrieved, even when reduced numbers of images are available. The TSC estimator hence provides a good trade-off between preserving the spatial resolution of interferograms and performing reliable phase statistics estimations when the number of SAR images is low.

Prior to the TSC evaluation, a sublook generation process should be carried out [2]. An unweighting filtering process is first performed in order to flatten the azimuth spectrum of the images. Then, the spectrum is split into two sublooks, which are consequently shifted to the same center frequency to avoid linear phase terms during the TSC evaluation. An inverse Fourier transform is finally applied to each sublook to obtain them in the spatial domain.

Since a pixel-wise product in the space-domain translates into a spectrum correlation in the frequency-domain, the TSC calculation is based on the use of the coherence estimator between temporal collections of sublooks as follows

$$
T S C=\frac{\sum_{t=1}^{N_{i m}} S L_{1}(t) \cdot S L_{2}^{*}(t)}{\sqrt{\sum_{t=1}^{N_{i m}}\left|S L_{1}(t)\right|^{2} \cdot \sum_{t=1}^{N_{i m}}\left|S L_{2}(t)\right|^{2}}}
$$

where $S L_{I}$ and $S L_{2}$ refer to the complex values from two pixels of the first and second sublook belonging to a SAR image acquired at instant $t$, and $N_{i m}$ refers to the total number of images available, i.e., the total number of looks involved in the estimation. Notice how the spatial averaging used in the traditional coherence approach is now replaced by a temporal one.

The pixels exhibiting high TSC amplitude values will be directly related to as point-like scatterers, and will be selected as potential candidates for the DInSAR processing. As shown later, due to the parallelism between the coherence estimator and the TSC approach, it is possible to extend this method to work with the Best [5], SOM [9], and ESM [10] polarimetric optimization methods.

\section{TSC POLARIMETRIC OPTIMIZATION}

\section{A. Best}

The simplest approach to improve the TSC estimation consists on selecting the polarimetric channel providing the highest value of TSC for the multi-temporal set of SAR images available. This method is referred to as Best [5]. Under this approach, the optimized $\mathrm{TSC}_{\mathrm{Best}}$ is given by

$$
\left|T S C_{B e s t}\right|=\max \left\{\left|T S C_{h h}\right|,\left|T S C_{h v}\right|,\left|T S C_{v v}\right|\right\}
$$

where the optimized interferometric phases will be addressed by the selected channel providing the highest value of TSC.

Thus, for each pair of acquisitions the original three interferograms available, i.e., one per each polarimetric channel, are reduced to a single one with the best response in terms of TSC. This is equivalent to select the best pixel in terms of phase quality. Nonetheless, this approach does not fully benefits from the potentials of polarimetry. The following methods, referred to as SOM [9] and ESM [10], make use of PolSAR data in a more efficient way.

\section{B. SOM}

Given an imaging geometry, the complex polarimetric scattering matrix $\mathbf{S}_{h v}$ gives information about the targets' scattering process within the illuminated scenario. Considering a monostatic configuration, $\mathbf{S}_{h v}$ is defined as follows

$$
\mathbf{S}_{h v}=\left[\begin{array}{ll}
S_{h h} & S_{h v} \\
S_{h v} & S_{v v}
\end{array}\right]
$$

where $S_{p q}$ accounts for the complex SAR images obtained from the receiving and transmitting electromagnetic waves at the polarization states $p$ and $q$, respectively. The horizontal and vertical polarization states are usually referred to as $p, q=h, v$.

In the context of this work, a scattering matrix may be defined for each sublook $i$ in the spatial domain as [11]

$$
\mathbf{S L}_{i, h v}(t)=\left[\begin{array}{ll}
S L_{i, h h}(t) & S L_{i, h v}(t) \\
S L_{i, h v}(t) & S L_{i, v v}(t)
\end{array}\right]
$$

where $i=1,2$ and $S L_{i, p q}$ accounts for the first and second complex spatial sublooks of an image obtained at the polarization states $p$ and $q$, at a certain instant $t$, respectively.

At this stage, the scattering matrix of each sublook $\mathbf{S L}_{i, h v}$ in the linear polarization basis $\{\mathbf{h}, \mathbf{v}\}$ may be expressed in any elliptical orthogonal basis $\{\hat{\mathbf{a}}, \mathbf{b}\}, \mathbf{S} \mathbf{L}_{i, a b}$, through the following unitary transformation [11]

$$
S L_{i, a b}(t)=\left[\begin{array}{cc}
S L_{i, a a}(t) & S L_{i, a b}(t) \\
S L_{i, a b}(t) & S L_{i, b b}(t)
\end{array}\right]=U_{2}^{T} \cdot S L_{i, h v}(t) \cdot U_{2}
$$

where ${ }^{T}$ indicates the vector transposition. At the same time, the transformation matrix $\mathbf{U}_{2}$ can be formulated as a function of the orientation and ellipticity angles $(\psi, \chi)$ of the polarization ellipse as follows [11]

$$
\mathbf{U}_{2}=\left[\begin{array}{cc}
\cos \psi & -\sin \psi \\
\sin \psi & \cos \psi
\end{array}\right]\left[\begin{array}{cc}
\cos \chi & j \sin \chi \\
j \sin \chi & \cos \chi
\end{array}\right]\left[\begin{array}{cc}
e^{+j \phi_{0}} & 0 \\
0 & e^{-j \phi_{0}}
\end{array}\right]
$$

where $\phi_{0}$ refers to an absolute phase term, which is irrelevant for DInSAR purposes, and is typically set to 0 .

Taking into account this theoretical description, the SOM approach consists on exploring all the range of orientation and ellipticity angles $(\psi, \chi)$ in order to find the basis transformation providing the highest TSC among all the co-polar $T S C_{a a}$ and cross-polar $T S C_{a b}$ values. Under this approach, the polarimetric optimized absolute value of the $\mathrm{TSC}_{\mathrm{SOM}}$ is given by [9]

$$
\left|T S C_{S O M}\right|=\max _{(\psi, \chi)}\left\{\left|T S C_{a a}(\psi, \chi)\right|,\left|T S C_{a b}(\psi, \chi)\right|\right\}
$$


where $T S C_{a a}$ and $T S C_{a b}$ may be expressed as

$$
\begin{aligned}
T S C_{a a} & =\frac{\sum_{t=1}^{N_{\text {im }}} S L_{1, a a}(t) \cdot S L_{2, a a}^{*}(t)}{\sqrt{\sum_{t=1}^{N_{\text {im }}}\left|S L_{1, a a}(t)\right|^{2} \cdot \sum_{t=1}^{N_{\text {im }}}\left|S L_{2, a a}^{*}(t)\right|^{2}}} \\
T S C_{a b} & =\frac{\sum_{t=1}^{N_{\text {im }}} S L_{1, a b}(t) \cdot S L_{2, a b}^{*}(t)}{\sqrt{\sum_{t=1}^{N_{\text {im }}}\left|S L_{1, a b}(t)\right|^{2} \cdot \sum_{t=1}^{N_{\text {im }}}\left|S L_{2, a b}^{*}(t)\right|^{2}}}
\end{aligned}
$$

and $S L_{\mathrm{i}, a a}$ and $S L_{\mathrm{i}, a b}$ refer to as the co-polar and cross-polar channels in the new $(\psi, \chi)$ polarization basis, for the temporal collections of the first and second sublook $i=1,2$, respectively.

The optimized interferometric phase is obtained through a simple image reconstruction from the optimized sublooks. All the spectrum transformations commented in Section II, these are the spectrum shift and weighting steps, are firstly undone. At this stage, the sublooks are joined in the spectrum domain leading to the optimized image through a simple inverse Fourier transform. The optimized interferogram for the later PolDInSAR processing is finally obtained from the phasedifferences of optimized SAR image pairs.

\section{ESM}

The SOM method improves the performance of the Best approach. Nonetheless, all the combinations generated by the different polarization basis constitute a subspace of the solutions generated by the ESM approach.

The scattering vector $\mathbf{k}_{\mathrm{i}}$ for each sublook $i$, may be defined as a vectorization of the sublook scattering matrix $\mathbf{S L}_{\mathrm{i}, \mathrm{hv}}$ [12]

$\mathbf{k}_{i, t}=\frac{1}{\sqrt{2}}\left[S L_{i, h h}(t)+S L_{i, v v}(t), S L_{i, h h}(t)-S L_{i, v v}(t), 2 S L_{i, h v}(t)\right]^{T}$

where $i=1,2$ indicates the two PolSAR sublooks from a SAR image acquired at a certain time $t$. From the entire possible basis to represent the scattering vector the Pauli basis is the most used. The so-called PolInSAR vector between two PolSAR sublooks' collections may be hence defined as [12]

$$
\mathbf{k}_{t}=\left[\mathbf{k}_{1, t}^{T}, \mathbf{k}_{2, t}^{T}\right]^{T},
$$

leading to the $6 \times 6$ PolInSAR sublook coherency complex matrix $\mathbf{T}_{6}[12]$

$$
\mathbf{T}_{6}=E\left\{\mathbf{k}_{t} \mathbf{k}_{t}^{H}\right\}=\left[\begin{array}{cc}
\mathbf{T}_{11} & \mathbf{\Omega}_{12} \\
\mathbf{\Omega}_{12}{ }^{H} & \mathbf{T}_{22}
\end{array}\right]
$$

where ${ }^{\mathrm{H}}$ refers to as the conjugate transpose, $T_{11}$ and $T_{22}$ correspond to the individual coherency matrices of each PolSAR sublook temporal collection, and $\Omega_{12}$ indicates the polarimetric interferometric coherency matrix between the sublook stacks. The expectation operator is replaced by a temporal averaging, in order to obtain sensitivity along the temporal axis, as seen in (1).

At this point, the TSC definition may be generalized taking into account its polarimetric dependence. The PolInSAR Pauli vector $\mathbf{k}_{i, t}$ for each sublook may be projected onto an unitary vector $\mathbf{w}$ in order to obtain a generic Sublook image $S L_{i}(t)=\mathbf{w}_{i}^{H} \mathbf{k}_{i, t}$. The generalized polarimetric $\mathrm{TSC}_{\mathrm{ESM}}$ for different combinations of $\mathbf{w}$ may be hence indicated as [12]

$$
\operatorname{TSC}_{E S M}(\mathbf{w})=\frac{\mathbf{w}^{H} \mathbf{\Omega}_{12} \mathbf{w}}{\sqrt{\mathbf{w}^{H} \mathbf{T}_{11} \mathbf{w} \mathbf{w}^{H} \mathbf{T}_{22} \mathbf{w}}}
$$

There are different methods available in the literature to assess the value of the optimum projection vector $\mathbf{w}$. The simplest one consists on parameterizing $\mathbf{w}$ as follows [12]

$$
\mathbf{w}=\left[\begin{array}{c}
\cos \alpha \\
\sin \alpha \cos \beta e^{j \delta} \\
\sin \alpha \sin \beta e^{j \gamma}
\end{array}\right] \quad\left\{\begin{array}{l}
0 \leq \alpha \leq \frac{\pi}{2} ; 0 \leq \beta \leq \pi \\
0 \leq \delta \leq \pi ;-\pi \leq \gamma<\pi
\end{array}\right.
$$

In this case, the optimization problem, which is solved by brute force, presents the main drawback of requiring a high computational cost. To overcome this limitation, the method presented in [10] by Colin, is proposed in this work. This approach is based on carrying out an iterative process assuming polarimetric stationarity, i.e., $\mathbf{T}_{11}$ and $\mathbf{T}_{\mathbf{2 2}}$ are similar. Under this assumption, the optimized $\mathrm{TSC}_{\mathrm{ESM}}$ is given by [10]

$$
T \hat{S} C_{E S M}(\mathbf{w})=\frac{\mathbf{w}^{H} \mathbf{\Omega}_{12} \mathbf{w}}{\mathbf{w}^{H} \mathbf{T} \mathbf{w}} \text { with } \mathbf{T}=\frac{\mathbf{T}_{11}+\mathbf{T}_{22}}{2}
$$

where $\left|T \hat{S}_{E S M}\right| \leq\left|T S C_{E S M}\right|$ is always accomplished [10]. With this numerical solution, the computational cost is drastically reduced up to three orders of magnitude. As in the previous case, the optimized interferometric phase is obtained through the image reconstruction from the optimized sublooks' collections.

\section{POLDINSAR RESULTS}

The results presented in this Section correspond to the measuring campaign carried out over the landslide of El Forn de Canillo, Andorra Pyrenees, see Fig. 1, from October 2010 to October 2011, using the X-band polarimetric GB-SAR sensor developed at the Universitat Politècnica de Catalunya (UPC), referred as RiskSAR-X [13]. In this framework, $10 \mathrm{X}$ band fully polarimetric acquisitions, with a temporal baseline of approximately one month, were collected.

The geological measurements collected during the last years have evidenced that the landslide of El Forn de Canillo is experiencing some residual movement between one and two centimeters per year [14]. In the North-East extreme of the main body of the landslide, a secondary slide, referred to as Cal Borró-Cal Ponet, reveals a higher activity coinciding with episodes of strong rainfalls [14]. 


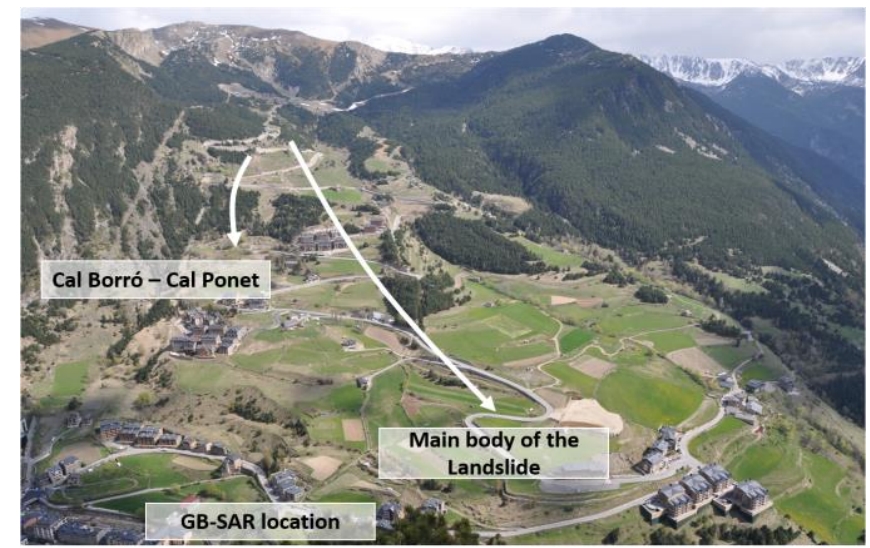

Fig.1. General overview of the landslide of El Forn de Canillo

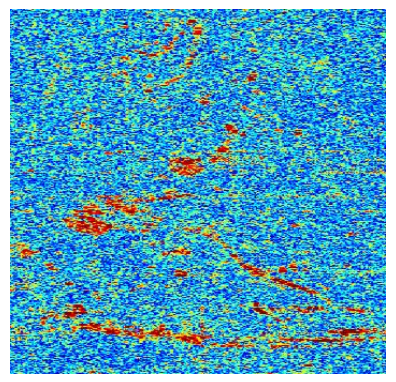

(a)

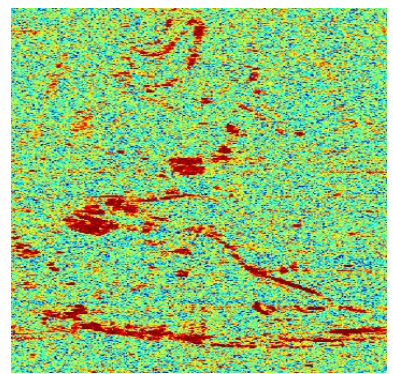

(c)

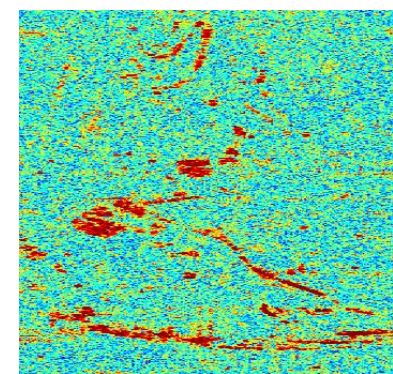

(b)

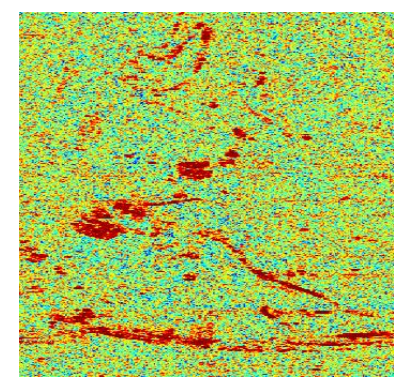

(d)
0

Fig.2. TSC map for (a) the single-polarimetric hh channel, and after applying the (b) Best, (c) SOM and (d) ESM polarimetric optimization approaches.

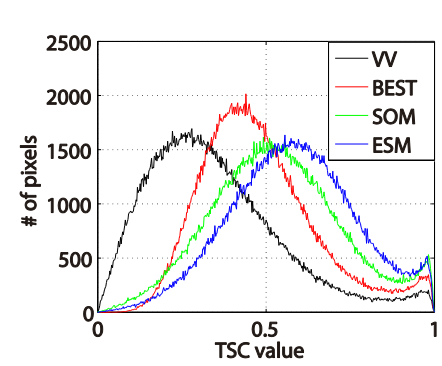

(a)

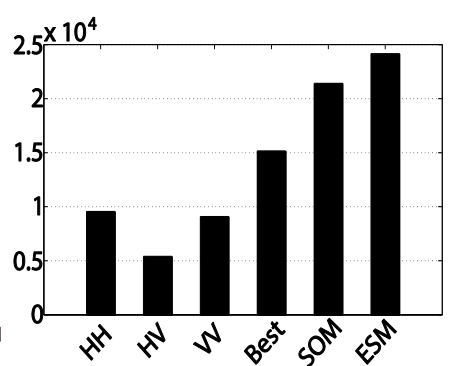

(b)
Fig.3 (a) TSC histograms for the single-polarimetric hh channel and after applying the Best, SOM, and ESM polarimetric optimization approaches. (b) Number of pixel candidates selected for each case.

El Forn de Canillo is mostly a vegetated area and the bare surfaces and man-made structures are scarce. In these scenarios, the potential density of persistent scatterers is expected to be very low, mainly due to the high temporal decorrelation present at X-band. On the one hand, the reduced number of images available, only ten, advises the use of the
TSC estimator. On the other hand, the reduced number of man-made structures in the scenario represents a perfect opportunity to check the benefits of applying polarimetric optimization methods in order to maximize the number of high-quality pixels' selected for the later DInSAR processing. Prior to carrying out the polarimetric optimization process, data must be correctly calibrated and the atmospheric artifacts must be correctly compensated for [15].

Fig. 2(a)-(d) show the amplitude TSC maps for the $h h$ single-polarimetric channel, and after applying the different polarimetric optimization methods presented. The comparison between the TSC histograms reveals that the ESM and SOM techniques produce the best response in terms of TSC, being the ESM approach slightly better, see Fig.3 (a). Notice how the TSC improvement is higher over the bare surfaces and low vegetated regions of the scenario. In the urban areas, which already present high TSC values for the single-polarimetric case, only a slight improvement is appreciated.

Indeed, this improvement in terms of phase quality is translated into higher densities of useful pixels during the DInSAR processing. In order to illustrate this, a TSC threshold of 0.85 , which corresponds to a phase standard deviation lower than $15^{\circ}$ [2], has been established during the pixel selection step. As seen in Fig. 3(b), up to more than a twofold increase in the number of pixel candidates is reached for this case.

Among the multiple DInSAR algorithms available in the literature, the Coherent Pixel Technique [16] has been employed to carry out the DInSAR processing. Fig. 4 shows the final deformation velocity maps geocoded over a Google Earth image for the $h h$ polarimetric channel, see Fig. 4 (a), and after applying the TSC ESM polarimetric optimization method, see Fig. 4 (b). Notice how the global behavior observed is similar for both methods, except for the increase of pixels' density. Results evidence that the main body of the landslide presents some residual movement of the order of 1$1.5 \mathrm{~cm}$ per year. In the top-left border of the main landslide, the secondary of Cal Ponet-Cal Borró is clearly identified with a motion rate of 2-2.5 $\mathrm{cm}$ per year. These results present a high agreement with the conclusions extracted from previous geological studies [14].

The displacement results over the same scenario employing the coherence stability approach were showed in [1]. Notice how the displacement magnitudes obtained in this work using the TSC approach reach slightly higher values. This difference is produced by the multi-looking carried out during the coherence estimation, which lead to an averaging of the displacement within the multi-looked area. For the same reason, the number of pixels selected with the TSC approach are significantly greater compared with the ones selected by the coherence stability approach. This fact benefits the construction of a more robust network during the minimization and integration processes of the CPT, thus benefiting the reliability of the DInSAR results. Despite these minor differences, the displacement results using the TSC approach present a high agreement with the ones presented in [1]. 


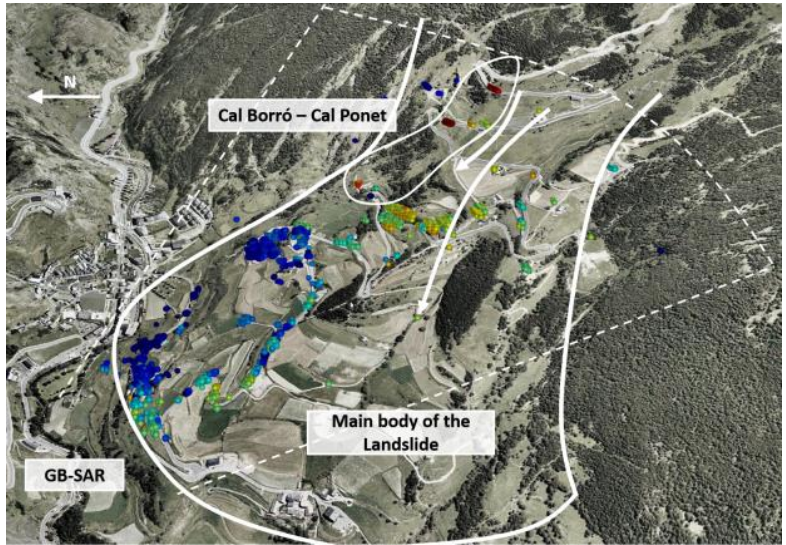

(a)

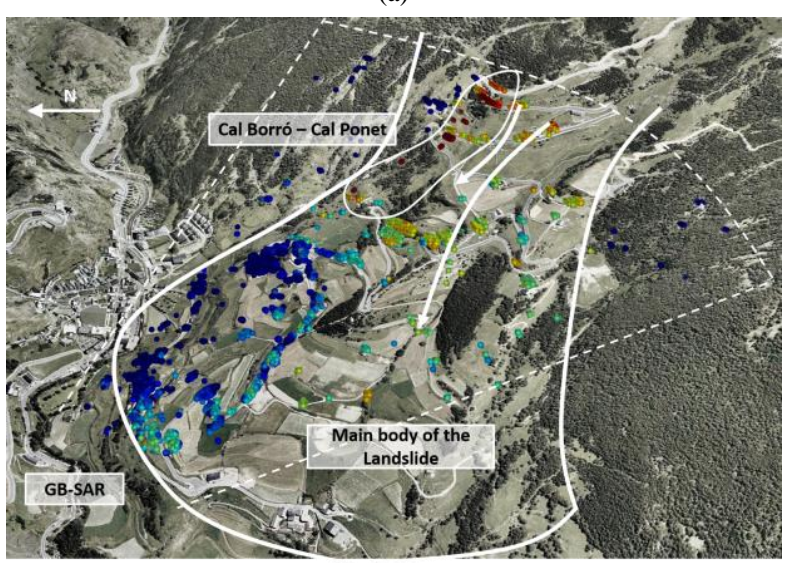

(b)

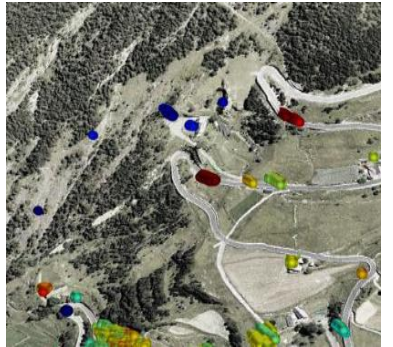

(c)

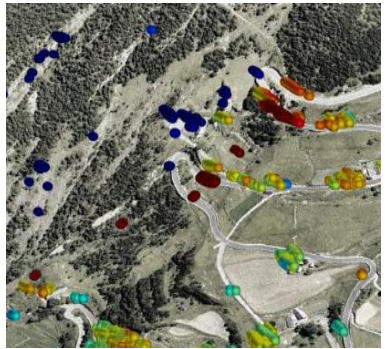

(d)
$0 \mathrm{~cm} /$ year

$2.5 \mathrm{~cm} /$ year

Fig.4. Lineal velocity retrieved using (a) the single-polarimetric hh channel and (b) the ESM TSC polarimetric optimization approach. Zoom of the area of Cal Ponet-Cal Borró for (c) the single-polarimetric hh channel and (d) the ESM TSC polarimetric optimization approach.

Finally, notice how the improvement achieved with the optimized ESM TSC polarimetric optimization approach in terms of pixels' density allows to better determine and characterize local phenomena. A clear example is the one produced in Cal Ponet-Cal Borró area, as it is illustrated in Fig. 4 (c) and (d).

\section{CONCLUSIONS}

In this paper, an extension of the TSC approach to work with the Best, ESM and SOM polarimetric optimization methods has been presented. The objective has been to improve the overall phase quality of the interferograms to be processed by means of DInSAR algorithms with the exploitation of fully- polarimetric data. The polarimetric optimized interferograms by means of TSC allowed the selection of a larger number of pixels, thus improving the displacement map retrieval process. The higher density and quality of the pixels selected help to better identify and characterize local phenomena, thus improving the geophysical understanding of such phenomena.

\section{REFERENCES}

[1] R. Iglesias, D. Monells, X. Fabregas, J. J. Mallorqui, A. Aguasca, and C. Lopez-Martinez, "Phase Quality Optimization in Polarimetric Differential SAR Interferometry," IEEE Trans. Geosci. Remote Sens., vol. 52, no. 5, pp. 2875-2888, May 2014.

[2] R. Iglesias, J. J. Mallorqui, and P. Lopez-Dekker, "DInSAR Pixel Selection Based on Sublook Spectral Correlation Along Time," IEEE Trans. Geosci. Remote Sens., vol. 52, no. 7, pp. 3788-3799, Jul. 2014.

[3] R. Lanari, O. Mora, M. Manunta, J. J. Mallorqui, P. Berardino, and E. Sansosti, "A small-baseline approach for investigating deformations on full-resolution differential SAR interferograms," IEEE Trans. Geosci. Remote Sens., vol. 42, no. 7, pp. 1377-1386, Jul. 2004.

[4] A. Ferretti, C. Prati, and F. Rocca, "Permanent Scatterers in SAR interferometry," IEEE Trans. Geosci. Remote Sens., vol. 39, no. 1, pp. 8-20, 2001.

[5] L. Pipia, X. Fabregas, A. Aguasca, C. Lopez-Martinez, S. Duque, J. J. Mallorqui, and J. Marturia, "Polarimetric Differential SAR Interferometry: First Results With Ground-Based Measurements," IEEE Geosci. Remote Sens. Lett., vol. 6, no. 1, pp. 167-171, Jan. 2009.

[6] V. D. Navarro-Sanchez, J. M. Lopez-Sanchez, and F. Vicente-Guijalba, "A Contribution of Polarimetry to Satellite Differential SAR Interferometry: Increasing the Number of Pixel Candidates," IEEE Geosci. Remote Sens. Lett., vol. 7, no. 2, pp. 276-280, Apr. 2010.

[7] V. D. Navarro-Sanchez and J. M. Lopez-Sanchez, "Improvement of Persistent-Scatterer Interferometry Performance by Means of a Polarimetric Optimization," IEEE Geosci. Remote Sens. Lett., vol. 9, no. 4, pp. 609-613, Jul. 2012.

[8] V. D. Navarro-Sanchez, J. M. Lopez-Sanchez, and L. Ferro-Famil, "Polarimetric Approaches for Persistent Scatterers Interferometry," IEEE Trans. Geosci. Remote Sens., vol. 52, no. 3, pp. 1667-1676, Mar. 2014.

[9] L. Sagues, J. M. Lopez-Sanchez, J. Fortuny, X. Fabregas, A. Broquetas, and A. J. Sieber, "Indoor experiments on polarimetric SAR interferometry," IEEE Trans. Geosci. Remote Sens., vol. 38, no. 2, pp. 671-684, Mar. 2000.

[10] E. Colin, C. Titin-Schnaider, and W. Tabbara, "An interferometric coherence optimization method in radar polarimetry for high-resolution imagery," IEEE Trans. Geosci. Remote Sens., vol. 44, no. 1, pp. 167175, Jan. 2006.

[11] J. Lee and E. Pottier, Polarimetric radar imaging: from basics to applications, CRC Press. CRC Press, 2009, p. 422.

[12] S. R. Cloude and K. P. Papathanassiou, "Polarimetric SAR interferometry," IEEE Trans. Geosci. Remote Sens., vol. 36, no. 5, pp. 1551-1565, 1998.

[13] A. Aguasca, A. Broquetas, J. J. Mallorqui, and X. Fabregas, "A solid state L to X-band flexible ground-based SAR system for continuous monitoring applications," in IEEE International IEEE International IEEE International Geoscience and Remote Sensing Symposium, 2004. IGARSS '04. Proceedings. 2004, 2004, vol. 2, no. C, pp. 757-760.

[14] J. Corominas, R. Iglesias, A. Aguasca, J. J. Mallorqui, X. Fagregas, X. Planas, and J. A. Gili, "Consideraciones sobre el deslizamiento del Forn de Canillo ( Princpipat d'Andorra) a partir de datos de Interferometría radar y nuevas obervaciones," in VIII Simposio Nacional sobre Taludes y Laderas Inestables, 2013, pp. 799-909.

[15] R. Iglesias, X. Fabregas, A. Aguasca, J. J. Mallorqui, C. LopezMartinez, J. A. Gili, and J. Corominas, "Atmospheric Phase Screen Compensation in Ground-Based SAR With a Multiple-Regression Model Over Mountainous Regions," IEEE Trans. Geosci. Remote Sens., vol. 52, no. 5, pp. 2436-2449, May 2014.

[16] P. Blanco-Sánchez, J. J. Mallorquí, S. Duque, and D. Monells, "The Coherent Pixels Technique (CPT): An Advanced DInSAR Technique for Nonlinear Deformation Monitoring," Pure Appl. Geophys., vol. 165, no. 6, pp. 1167-1193, Aug. 2008. 